mäßig angenommen werden kann oder welchen mittleren Wert man dafür unter verschiedenen Verhältnissen in Gleichung (I) einsetzen muß. Eine weitere experimentelle Aufgabe ist die Bestimmung des mittleren Wärmeleitvermögens $k$ von Wicklungen. Die Größe $\mathrm{k}$ ist zwar bei der Berechnung von $\mathrm{c}$ entbehrlich, nicht aber für die Vorausberechnung der Absolutwerte der Temperaturen. k kann entweder nach einer der bekannten Methoden zur Bestimmung des Wärmeleitvermögens gemessen werden oder z. B., indem man eine Wicklung von rechteckigem Querschnitt durch ihren eigenen Strom heizt, dabei ihre Oberflächentemperatur überall gleich hält (etwa durch Lagerung in einem gut gerührten Ölbad) und $t_{\text {m }}$ aus der Widerstandszunahme bestimmt. $\mathrm{k}$ läßt sich dann aus Gleichung (2 I) berechnen, ohne daß man im Innern der Spule ein Thermoelement anbringen müßte ${ }^{1}$ ).

\title{
Zur Frage der Messung von Oberflächentemperaturen in der Elektrotechnik.
}

\author{
Von \\ Max Jakob. \\ (Mitteilung aus der Physikalisch-Technischen Reichsanstalt.)
}

I. Bedeutung der Messung von Oberflächentemperaturen für die Elektrotechnik. Nach den vom Verband deutscher Elektrotechniker herausgegebenen Normalien wird bisher die Temperaturerhöhung der Transformatorenwicklungen, der mit Gleichstrom erregten Feldspulen und aller ruhenden Wicklungen von Generatoren und Motoren aus der Widerstandszunahme bestimmt, die Temperatur aller anderen Teile von Maschinen mittels Thermometern ${ }^{2}$ ). Nun hat neuerdings $\mathrm{Vidmar}{ }^{3}$ ) den bemerkenswerten Vorschlag gemacht, Widerstandszunahme u n d Oberflächentemperatur der Wicklungen $\mathrm{zu}$ messen. Es besteht nämlich żwischen der höchsten Temperatur $t_{i}$, der mittleren Temperatur $t_{w}$ und der Oberflächentemperatur $t_{o}$ einer Wicklung, wenn $t_{o}$ überall gleich ist, die Beziehung

$$
t_{i}=a t_{w}+(1-a) t_{0} \text {. }
$$

Dabei ist $a=\frac{t_{i}-t_{0}}{t_{w}-t_{o}}$ und schwankt nach den Berechnungen von $\left.\left.O t t^{4}\right), V i d m a r^{3}\right)$, Rogows ki ${ }^{5}$ ) nur in engen Grenzen, nämlich für Spulen beliebiger Form zwischen $I, 5$ und 2,5, für hohlzylindrische Spulen von nicht zu geringer lichter Weite, wie ich vor kurzem gezeigt habe ${ }^{6}$ ), zwischen I,5 (bei unendlicher Spulenlänge) und 2, I (bei quadratischem Querschnitt). Vidmar hat vorgeschlagen, $\mathrm{a}=2 \mathrm{zu}$ setzen und die zulässigen Höchstwerte von $t_{i}$ in den Verbandsnormalien festzulegen. $t_{w}$ wäre dann durch Widerstandsmessung, $t_{0}$ thermometrisch zu bestimmen.

Wird Vidmars Vorschlag prinzipiell angenommen, so wird die Messung von Oberfiächentemperaturen in der Elektrotechnik künftig eine viel größere Rolle

1) Bei dieser Meßmethode wie bei allen obigen Berechnungen ist $\mathrm{k}$ als von der Tem. peratur unabhängig angenommen, was in dem bei Wicklungen höchstmöglichen Temperaturintervall von etwa $\operatorname{Io0}^{\circ}$ und gemäß der geringen beanspruchten Genauigkeit durchaus zulässig ist.

2) Normalien für Bewertung und Prüfung von elektrischen Maschinen und Transformatoren $\$ \$ 15$ bis 17 . I9I3.

3) M. Vidmar, Elektrotechnik und Maschinenbau 36. S. 49, 64. I918.

) L. Ott, Mitt. über Forschungsarbeiten auf dem Gebiete des Ingenieurwesens. Heft 35 und 36. S. 53. rgo6.

5) W. Rogowski, Archiv für Elektrotechnik 7. S. 4I. Igr8.

6) M. J akob, Archiv für Elektrotechnik, 8. S. 1x7. I9rg. 
spielen als bisher. Gemäß Gleichung (I) wird nämlich $t_{0}$, je nach der Wahl von a, mit vollem oder annähernd vollem Betrag in die Messung der Höchsttemperatur $t_{i}$ eingehen, also für die Beurteilung der Erwärmung jeder Wicklung maßgebend sein. Dies gibt mir Veranlassung zu einigen Bemerkungen.

2. Messung von Oberflächentemperaturen nach den Vorschriften der Verbandsnormalien. Über die Messung von Oberflächentemperaturen heißt es in $\S$ I4 der Verbandsnormalien: „Wird ein Thermometer zum Messen der Temperatur verwendet, so muß für eine möglichst gute Wärmeleitung zwischen diesem und dem zu messenden Maschinenteil gesorgt werden, z. B. durch Stanniolumhüllung. Zum Vermeiden von Wärmeverlusten wird außerdem die Kugel des Thermometers und die Meßstelle gemeinsam mit einem schlechten Wärmeleiter (trockener Putzwolle u. dgl.) überdeckt." Es soll nun an Hand einiger überschlägigen Rechnungen ') gezeigt werden, daß auch die genaue Befolgung dieser Vorschrift nicht vor beträchtlichen Meßfehlern schützt. Diesen Berechnungen, zu deren Vereinfachün als $\mathrm{Null}$ punkt der Temperaturmessung durchweg die Temperatur der Umgebung angenommen werden möge, lege ich folgende Verhältnisse zugrunde (s. Abb. I):

Auf ein Stück $f$ der Oberfläche, deren Temperatur zu messen ist (im folgenden der Einfachheit halber ,Meßfläche“ genannt), möge das Thermometer, nach Vorschrift des $\$ 14$ der Verbandsnormalien umhüllt, aufgesetzt werden. Der das Thermometer umgebende gute Wärmeleiter (Stanniol) liege ohne den geringsten Luft$z w i s c h e n r a u m$ an der Fläche $f$ und an der Thermomèterkugel auf. Sein Wärmeleitvermögen sei so groß, daß man annehmen darf, er habe im Dauerzustande überall die gleiche Temperatur wie die

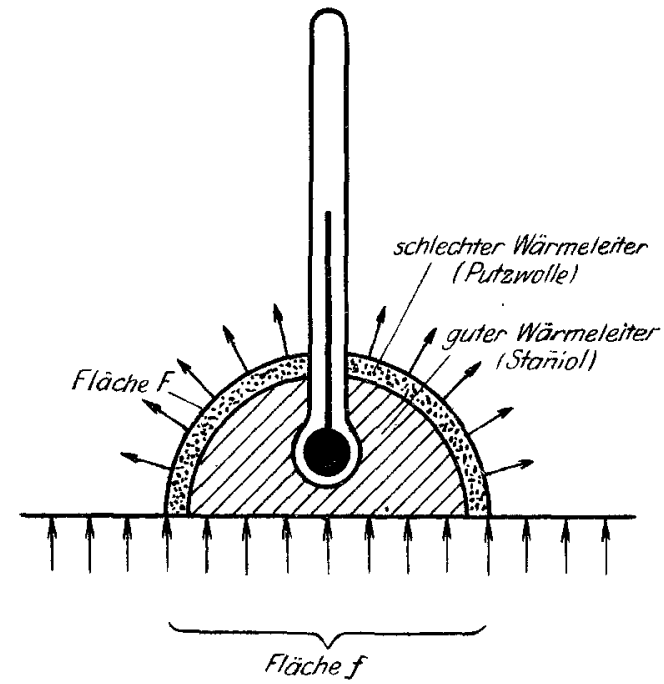

Abb. I. Anordnung eines Quecksilberthermometers zur Messung der Oberflächentemperatur.

Fläche f. Diese Temperatur werde vom Thermometer fehlerfrei angezeigt. Die Dicke der den guten Wärmeleiter abdeckenden Isolierschicht (Putzwolle) sei d, ihr Wärmeleitvermögen ${ }^{2}$ ) $\lambda$.

Vor dem Aufsetzen des Thermometers und seiner Hülle möge durch $f$ die Energie W [Watt] an die Umgebungsluft übergegangen sein; bei einem äußeren Wärmeleitvermögen ${ }^{3}$ ) $\alpha$ sei hierbei $t_{o}$, die Temperatur, der Fläche f gewesen. Durch Aufsetzen des Thermometers mit Hülle wird der Betrag der die Fläche $\mathrm{f}$ durchsetzenden Energie verändert. Er sei nun $W^{\prime}$ und gehe durch die Oberfläche $F$ der Thermometerhülle an die Luft über ${ }^{4}$ ). Die Fläche $F$ habe das äußere Wärmeleitvermögen $a^{\prime}$ und nehme im Dauerzustande der Wärmeströmung die Temperatur $t_{o}^{\prime}$ an. Infolge der Veränderung der Energieströmung ändert sich natürlich auch die Temperatur der Fläche $f$; sie gehe von $t_{0}$ in den Wert $t$ über. Letztere Temperatur wird von dem Thermometer angezeigt.

1) Ähnliche Berechnungen sind enthalten in dem Buch von Osc. Knoblauch und K. $\mathrm{H}$ encky, ,Anleitung zu genauen technischen Temperaturmessungen mit Flüssigkeits- und elektrischen Thermometern", München u. Berlin I9I9, R. Oldenburg.

2) In der Technik auch „Wärmeleitzahl“ genannt.

3) In der Technik auch "Wärmeübergangszahl" genannt.

4) Der durch den Quecksilberfaden und das Thermometerglas abströmende Energiebetrag sei dabei mit eingerechnet. 
Nun ist offenbar für:

$$
W^{\prime} \gtreqless W \quad t \lesseqgtr t_{0}
$$

Denn bei größerer Wärmeentziehung aus dem Körper, dessen Oberflächentemperatur $t_{0}$ zu messen ist, bildet sich in dessen Innern ein stärkeres Temperaturgefälle aus und umgekehrt.

Ferner gelten näherungsweise ${ }^{1}$ ) folgende Beziehungen :

$$
\begin{aligned}
& W=\mathrm{f} \cdot \alpha \cdot \mathrm{t}_{0} \\
& \mathrm{~W}^{\prime}=\mathrm{F} \cdot \alpha^{\prime} \cdot \mathrm{t}_{0}^{\prime} \\
& \mathrm{W}^{\prime}=\frac{\mathrm{F}}{\mathrm{d}} \cdot \lambda \cdot\left(\mathrm{t}-\mathrm{t}_{\mathrm{o}}{ }^{\prime}\right)
\end{aligned}
$$

Aus (4) und (5) folgt

$$
t=\frac{W^{\prime}}{F \alpha^{\prime}}\left(1+\frac{\alpha^{\prime} d}{\lambda}\right)
$$

und durch Multiplikation mit Gleichung (3)

$$
t=\frac{f \alpha}{\mathrm{F} \alpha^{\prime}} \cdot \frac{W^{\prime}}{W}\left(i+\frac{\alpha^{\prime} \mathrm{d}}{\lambda}\right) t_{0} .
$$

Aus Gleichung (2) und (6) geht hervor, daß nur dann $t=t_{0}$ ist, also eine nach Vorschrift des § I4 der Verbandsvorschriften vorgenommenen Messung fehlerfrei, wenn

$$
\frac{\mathrm{f} \alpha}{\mathrm{F} \alpha^{\prime}}\left(\mathrm{I}+\frac{\alpha^{\prime} \cdot \mathrm{d}}{\lambda}\right)=\mathrm{I}
$$

Diese Bedingungsgleichung wird aber nur zufällig einmal erfüllt sein. Ist sie nicht erfüllt, so ist $t \geqslant t_{0}$, die Messung also mit einem Fehler behaftet. Wie groß dieser Fehler ist, läßt sich nicht allgemein berechnen; wohl aber läßt sich $t$ durch Beachtung der Gleichung (z) zwischen eine obere und eine untere Grenze einschließen Ist nämlich

$$
\frac{\mathrm{f} \alpha}{\mathrm{F} \alpha^{\prime}} \quad \frac{\mathrm{W}^{\prime}}{\mathrm{W}}\left(\mathrm{I}+\alpha^{\prime} \frac{\mathrm{d}}{\lambda}\right) \gtrless \mathrm{I}
$$

so ist nach Gleichung (6)

$$
\begin{aligned}
t & \geqslant t_{0} \\
w^{\prime} & \geqslant w .
\end{aligned}
$$

und nach Gleichung (2)

(6) ein, so erhält man den einen Grenzwert;

Setzt man also $\frac{W^{\prime}}{W}=I$ in Gleichung (6) ein, so erh
$\mathrm{t}=\mathrm{t}_{\mathrm{o}}$ ist der andere Grenzwert. In Gleichungsform:

$$
\mathrm{t}_{\mathrm{o}} \leqslant \mathrm{t} \leqslant \frac{\mathrm{f} \alpha}{\mathrm{F} \alpha^{\prime}}\left(\mathrm{I}+\alpha^{\prime} \frac{\mathrm{d}}{\lambda}\right) \mathrm{t}_{\mathrm{o}}
$$

Beispiele: Nach Abb. I sei z. B. die Meßfläche kreisförmig, also $f=r^{2} \pi$, die Oberfläche der Thermometerhülle halbkugelförmig, also $F=2 r^{2} \pi$, somit $f / F=1 / 2$. Ferner sei das Wärmeleitvermögen von Putzwolle $\lambda=0,6 \cdot 10^{-3}\left[\frac{\text { Watt }}{\mathrm{cm} \mathrm{Grad}}\right] \cdot$ Diese Annahmen mögen für die folgenden Beispiele $1-6$ gemeinsam gelten.

Beispiel I: Die Oberfläche, deren Temperatur gemessen werden soll, ruhe und sei nicht künstlich ventiliert. Dabei sei $\alpha=0,7 \cdot 10^{-3}\left[\frac{\text { Watt }}{\mathrm{cm}^{2} \mathrm{Grad}}\right]$.

1) Die Gleichungen (3) und (4) (bekanntlich von Newt on stammend) sind nur in erster Annäherung richtig. Gleichung (5) gilt nur für eine im Verhältnis zur Krümmung der Oberfläche $\mathrm{F}$ geringe Dicke d. Für die folgenden überschlägigen Rechnungen sind die Gleichungen "genau genug. 
Durch das Aufsetzen des Thermometers auf die Fläche $\mathrm{f}$ werde die Wärmeübergangszahl nicht verändert, es sei also $\alpha=\alpha^{\prime}$. Durch Einsetzen der Zahlenwerte in Gleichung ( 6 a) folgt dann $\mathrm{d}=0,86 \mathrm{~cm}$. Unter den obigen Voraussetzungen wäre die Messung also nur bei einer Putzwollschicht von $0,86 \mathrm{~cm}$ Dicke richtig, bei jeder anderen Dicke mehr oder weniger fehlerhaft.

Beispiel 2: Wenn unter den Verhältnissen des I. Beispiels die Dicke der Putzwollschicht $d=0,5 \mathrm{~cm}$ genommen würde, so wäre nach Gleichung $(6 \mathrm{~b})$

$$
t_{0}>t>0,79 t_{0} \text {. }
$$

Der Mittelwert der Grenzwerte wird dem wirklich gemessenen Wert nahe kommen. Es ist also annähernd $t=0,895 t_{0}$. Man hätte dann etwa $10 \%$ zu geringe Temperatur gemessen.

Beispiel 3: Bei einer Putzwollschicht von der Dicke $d=1,5 \mathrm{~cm}$ wäre nach Gleichung $(6 b)$ unter den Verhältnissen des I. Beispiels

$$
t_{0}<t<r, 3>t_{0}
$$

Analog wie beim Beispiel 2 kann man wohl annehmen $t \doteq 1,185 t_{0}$. Die Messung gäbe also wahrscheinlich um etwa. I $8 \% \mathrm{zu}$ hohe Temperatur.

Beispiel 4: Wäre $z$ war $d=0,86 \mathrm{~cm}$, wie im Beispiel I berechnet, aber $\alpha^{\prime}$ etwas verschieden von $\alpha, z$. B. $\alpha^{\prime}=\mathrm{I}, 2 \alpha=0,84 \cdot 1 \mathrm{O}^{-3}$, so wäre

also der Meßfehler etwa $-4 \%$.

$$
t_{0}>t>0,92 t_{0}
$$

Beispiel 5: Wäre zwar $\alpha=\alpha^{\prime}$, handelte es sich jedoch um die Messung der Temperatur einer durch einen Luftstrom von etwa $20 \mathrm{~m} / \mathrm{sk}$ Geschwindigkeit ventilierten ruhenden Fläche, wobei etwa $\alpha=\alpha^{\prime}=0,3 \cdot \mathrm{IO}^{-3}$ wäre, so könnte nach Gleichung ( $6 \mathrm{a}$ ) die Messung nur richtig sein, wenn $\mathrm{d}=2,0 \mathrm{~cm}$ wäre. Würde man $\mathrm{d}=0,86 \mathrm{~cm}$ beibehalten, so müßte man sich nach Gleichung (6b) auf einen Meßfehler von etwa - $14 \%$ gefaßt machen.

Beispiel 6: Wäre die Temperatur eines schnell rotierenden und somit stark ventilierten Maschinenteils nach $\mathrm{Ab}$ stellen der Maschine zu messen, so wäre etwa $\alpha=3,0 \cdot 10^{-3}$, $a^{\prime}=0,7 \cdot 10^{-3}$ anzunehmen. Die Gleichung (6a) würde in diesem Falle zu einem negativen Wert von d führen. Unter den angenommenen Verhältnissen wäre also eine richtige Messung der Temperatur überhaupt unmöglich. Das größte äußere Wärmeleitvermögen, für welches eine richtige Messung möglich wäre, ist $\boldsymbol{\alpha}=\mathrm{I}, 4 \cdot \mathrm{IO}^{-3}$. Nach Gleichung (6a) müßte dann $\mathrm{d}=\mathrm{o}$ sein: Man würde also in diesem Fall richtig messen, wenn man die Putzwollumhüllung gänzlich wegließe.

Nach der Fassung des $\S$ I 4 der Verbandsnormalien lag für die Praxis bisher natürlich keine Veranlassung vor, auf die Dicke der Putzwollschicht, auf den Wert der Verhältnisse $f / F$, $\alpha / \alpha^{\prime}$ und $\alpha^{\prime} / \lambda \mathrm{zu}$ achten. Es sind daher zweifellos Meßfehler

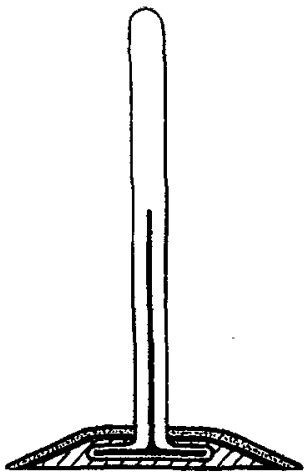

Abb. 2. Flache An. ordnung eines Quecksilberthermometers zur Messung von Oberflächentemperaturen. von 10,20 und mehr $\%$ das Übliche gewesen. Bliebe dies auch künftig, so wäre der durch den eingangs erwähnten Vorschlag von Vidmar angestrebte Fortschritt durchaus illusorisch.

\section{Brauchbare Verfahren zur Messung von Oberflächentemperaturen.} Man könnte daran denken, eine Messanordnung für Quecksilberthermometer nach Abb. I auszubilden und dabei die Dicke der Isolierschicht d ein für allemal.so zu wählen, daß bei natürlicher Konvektion die Bedingungsgleichung (6a) erfüllt wäre. Dabei würde sich die Verwendung eines flachen Quecksilbergefäßes und möglichst flache Anordnung, etwa nach Abb. 2, empfehlen, weil hierbei $\mathrm{f} / \mathrm{F}$ und $\alpha / \alpha^{\prime}$ nicht zu sehr vom Wert I abweichen. Der das Quecksilbergefäß umschließende gute Wärmeleiter (Stanniol, Kupferpulver o dgl.) wäre zweckniäßig durch ein dünnes 
Kupferblech allseitig zu umschließen. Die Isolierschicht müßte aus einem homogenen Stoff von möglichst geringem $\lambda$ bestehen. Die Oberfläche $F$ sollte in Farbe und Gefüge tunlichst der Meßfäche f gleichen. Zur Messung an einem Kollektor wäre also die Isolierschicht mit einer Kupferfolie zu bekleiden. Die Dicke d der Isolierschicht müßte experimentell festgestellt werden ${ }^{1}$ ); zur Messung der Temperatur ventilierter Oberflächen wäre sie entsprechend zu verändern. Die Bestimmung von d wäre natürlich Aufgabe des Herstellers einer derartigen Oberflächenthermometeranordnung. Dem Elektrotechniker müßte diese zusammengebaut mit den nötigen Anweisungen und Eichdaten für seine praktischen Messungen übergeben werden. Die Justierung, Eichung und Handhabung eines solchen Instrumentes würde natürlich beträchtliche Sorgfalt erfordern ${ }^{2}$ ).

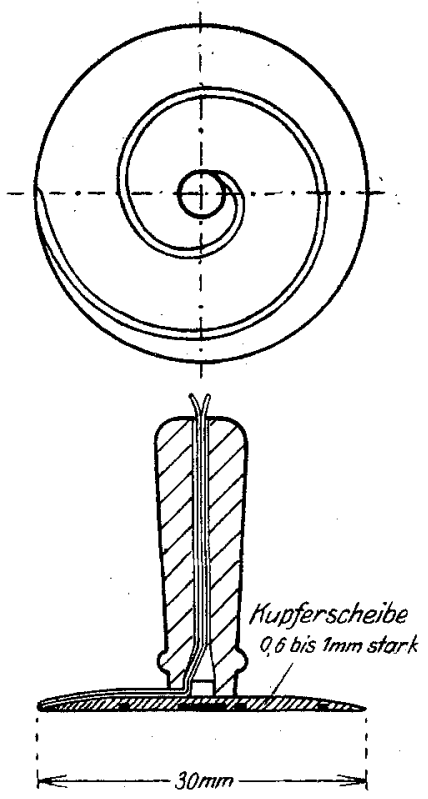

Abb. 3. Oberflächenthermoelement nach Hencky.

Viel einfacher gestaltet sich die Oberflächentemperaturmessung mit Thermoelementen. In der Starkstromtechnik sind diese zwar aus naheliegenden Gründen nicht beliebt. Ihre Anwendung wird aber künftig schwerlich zu vermeiden sein und ist jedenfalls möglich, da die Messungen im allgemeinen nach Abstellen der Maschinen, also ohne Gefahr von Überschlägen und Induktionseinflüssen ${ }^{3}$ ) stattfinden. Temperaturdauermessungen . an laufenden oder erregten Maschinen bezwecken meistens nur festzustellen, wann der stationäre Wärmezustand erreicht ist ; diese Feststellung läßt sich nötigenfalls nach wie vor mit Quecksilberthermometern machen, ohne daß auf deren Anordnung die Sorgfalt verwendet werden müßte, die für richtige Messung der Temperaturen erforderlich wäre. In Öltransformatoren endlich wird die Oberflächentemperatur der Spulen durch Messung der Öltemperatur mit Quecksilberthermometern in vielen Fällen genügend genau zu bestimmen sein.

Auch bei Messung der Oberflächentemperatur mit Thermoelementen sind wichtige Regeln zu beachten, wenn grobe Meßfehler vermieden werden sollen. Vor allem darf man sich nicht damit begnügen, die Lötstelle

des Thermoelements an die Meßfläche anzupressen ${ }^{4}$ ) und die Drähte des Elements unmittelbar hinter der Lötstelle von der Oberfläche wegzuführen. Dabei würde man infolge der Wärmeableitung durch die Drähte die Temperatur um viele Grade zu niedrig messen. Auch die Anwendung sehr dünner Drähte würde nichts verbessern, da die Temperatursenkung an der Lötstelle, wie Nusselt ${ }^{5}$ ) nachgewiesen hat, vom Drahtdurchmesser unabhängig ist.

1) Für diesen Zweck gut brauchbare Eichvorrichtungen hat K. Henck y im "Gesundheitsngenieur" 41. S. 95. 1918 beschrieben.

2) Auf Grund ähnlicher Berechnungen, wie der zu Gleichung (6) und (6a) führenden, hat W. van Rinsum (Z. d. V. d. J. 62. S. 604-605. 1918) ein Oberflächenthermoelement angegeben, bei dem, wie oben durch die Wahl der Dicke d der Isolierschicht, durch Verănderung der Wärme abgebenden Oberfläche mittels eines Schiebers eine „Abstimmung“ des Meßinstrumentes erfolgt. Einfacher als dieses Thermoelement sind die im folgenden beschriebenen Anordnungen. schädlich.

3) Kleinere Induktionseinflüsse sind infolge der bifilaren Bauart der Thermoelemente un-

4) Nach dem im 2. Abschnitt Gesagten ist selbstverständlich sowohl eine Isolierschicht über der Lötstelle, als überhaupt eine Vergrößerung der ableitender. Oberflächen zu vermeiden. Man wird die Lötstelle vielmehr etwa mit Bindfaden fest anbinden oder in ähnlicher Weise befestigen. Siehe auch S. I3I, Fußnote 3 .

5) W. Nusselt, Mitteil. über Forschungsarbeiten auf dem Gebiete des Ingenieurwesens. Heft 63 und 64 . Seite $24-28$. 1909. 
Man muß daher die Thermoelementendrähte von der Lötstelle ab einige $\mathrm{cm}$ (etwa IO $\mathrm{cm}$ ) durch ein Gebiet gleicher Temperatur, also am besten auf der Oberfläche weiterführen. Bei Spulen wird man etwa zweckmäßigerweise die Drähte in der Wicklungsrichtung einmal herumschlingen, wenn dies möglich ist. Die Drähte müssen aber eng an der Oberfläche anliegen.

Dies Prinzip der Messung läßt sich auf eine kleine Oberfläche ůbertragen, indem man, von der Lötstelle ausgehend, die Drähte in eine ebene Spirale aufwickelt und diese Spirale nebst der Lötstelle auf die Meßfäche aufpreßt. Nach Knoblauch und $\mathrm{Hencky}^{1}$ ) „füllt man den Zwischenraum zwischen den Spiralen mit Metallpulver aus, welches man des besseren Haftens wegen etwas mit Öl benetzt hat. Das Ganze wird sodann mit einem dünnen Plättchen fest an die Fläche gepreßt". Des letzteren Oberfläche muß wieder in Farbe und Gefüge der Meßfläche ähneln. „Für vertikale Flächen stattet man das Plättchen mit einem kleinen Bord aus, welcher das Herausfallen des Pulvers verhindert." Eine solche Spirale läßt sich leicht gekrümmten Flächen anschmiegen.

Dies ist nicht ganz so leicht möglich bei dem folgenden von $\mathrm{Hencky}{ }^{2}$ ) angegebenen Oberflächenthermoelement, welches aber im übrigen wohl als das vollendetste Instrument dieser Art bezeichnet werden darf. Es besteht aus einem nur 0,6 bis I mm starken Kupferplättchen von $30 \mathrm{~mm}$ Durchmesser mit einer spiraligen Nut (s. Abb. 3), in welcher die Thermoelementendrähte von der mittleren Lötstelle nach außen geführt werden. Das Plättchen wird entweder durch einen kleinen Griff aus Isoliermaterial oder, wenn man auch den kleinen Metallansatz zu seiner Befestigung am Kupferplättchen vermeiden will, durch Ebonitstäbchen an die Fläche angepreß ${ }^{3}{ }^{3}$ ), deren Temperatur zu bestimmen ist. Die äußere Oberfläche wird wieder möglichst ähnlich der Meßfäche ausgebildet. Bei richtiger Anwendung dieses Oberflächenthermoelements ist die in der elektrotechnischen Praxis erwünschte Meßgenauigkeit leicht $\mathrm{zu}$ erreichen.

Es sei noch erwähnt, daß man die Oberflächentemperatur natürlich auch durch Messung der Widerstandszunahme einer dünnen auf die Oberfäche aufzupressenden Wicklung bestimmen kann, bezüglich deren Anordnung und Anwendung ähnliche Vorsichtsmaßregeln nötig sind, wie bei den Thermoelementen. Aus der Widerstandszunahme einer um eine Spule eng herumgeschlungenen Wicklung von einigen Drähten z. B. kann man die Temperatur der Oberfläche bequem und genügend genau ermitteln. Kleineren Induktionseinflüssen kann durch bifilare Anordnung begegnet werden.

Hingewiesen werden soll endlich noch darauf, daß beim Abstellen von Maschinen, wie bekannt, je nach den Verhältnissen bald schnelle Abkühlung, bald Erwärmung durch Wärmestauung (infolge der geringen Wärmeabgabe an die Luft) eintreten kann. Vielfach wird nun in der elektrotechnischen Praxis mit dem Ablesen des Thermometers gewartet, bis es seinen Höchstwert erreicht hat. Man kann dann unter Umständen die Oberflächentemperatur zu hoch messen. Mit Henckys Oberflächenthermoelement dürfte bei Messung auf Metall nach I bis 2 Minuten, bei Messung auf isolierten Wicklungen nach 5 bis Io Minuten der Temperaturausgleich zwischen Oberfläche und Thermoelement fașt völlig erreicht sein ${ }^{4}$ ). Wesentliche

1) Osc. Knoblauch und K. Hencky, a. a. O. S. 89.

2) K. Hencky, Zeitschr. für die gesamte Kălteindustrie 22, S. 8I bis 84, 1915. Derselbe, Gesundheitsingenieur 41. S. gr bis 97. 1918 .

$\left.{ }^{3}\right)$ Der Temperaturausgleich durch die Kupferscheibe bewirkt, daß die Störung der Wärme. übergangsverhältnisse durch den Griff keinen wesentlichen Einfluß auf die Messung hat. Eine einfache Lötstelle dürfte man nicht durch einen Griff anpressen.

$\left.{ }^{4}\right) \mathrm{K} . \mathrm{Hencky}$, a. a. O. 
Temperatursteigerungen nach 2 bzw. Io Minuten Wartezeit sind dann auf Wärmestauung zurückzuführen ${ }^{1}$ ).

4. Ergebnis der Untersuchung. Durch den Vorschlag von Vidmar, die zulässigen Höchsttemperaturen der Wicklungen in den Verbandsnormalien festzulegen und sie nach Gleichung (I) aus Widerstandszunahme und Oberflächentemperatur zu bestimmen, ist, wie in Abschnitt I dargelegt ist, die Frage der Messung von Oberflächentemperaturen für die Elektrotechnik besonders aktuell und wichtig geworden. Das Vorgehen nach den Vorschriften des \& I4 der Verbandsnormalien führt nach den überschlägigen Berechnungen und einfachen Anwendungsbeispielen des 2. Abschnitts im allgemeinen zu unzulässig großen Fehlern der Temperaturmessung. Nur wenn man die Dimensionen der isolierenden Umhüllung der Quecksilberthermometer so wählt, daß die Bedingungsgleichung (6a) erfüllt ist, kann man ein richtiges Resultat erwarten. Im Abschnitt 3 wird zunäçst angegeben, wie demnach ein Quecksilberthermometer zweckmäßig anzuordnen wäre, dann aber ausgeführt, daß die Messung mit Thermoelementen viel einfacher und bequemer ist. Es wird gezeigt, durch welche Maßnahmen hierbei grobe Meßfehler vermieden werden und welche Formen von Oberflächenthermoelementen für die Zwecke der Elektrotechnik als besenders geeignet erscheinen.

Auf Grund der vorliegenden Ausführungen wird hiermit angeregt, daß der \& I4 der Verbandsnormalien abgeändert werde und daß entweder in den Normalien selbst oder in den Erläuterungen dazu genaue Direktiven für die Messung von Oberflächentemperaturen nach dem heutigen Stand der Thermometrie gegeben werden mögen. Nur so kann der erwähnte Vorschlag Vidmars wirklich zu einem Fortschritt in der-Bewertung und Prüfung elektrischer Maschinen führen.

\section{Zur Theorie des Parallelbetriebes von Synchronmaschinen.}

\section{Von L. Dreyfus.}

Wenngleich über den Parallelbetrieb von Synchronmaschinen eine Reihe vorzüglicher Arbeiten erschienen ist, so kann man doch kaum behaupten, daß alle Seiten dieses schwierigen Problems bereits geklärt seien. Man braucht nur daran zu denken, daß sich mit Ausnahme einer Arbeit von Duffing ${ }^{2}$ ) alle Autoren auf das Studium kleiner Schwingungen beschränken, um die analytische Behandlung zu vereinfachen. Ḋer Einfluß der Eisensättigung und der Netzbelastung auf die Konstanten der Pendelung sind gleichfalls noch nicht, oder noch nicht genügend untersucht. Auch ist es noch nicht gelungen, die Größse der elektrischen Dämpfung, mit welcher die mechanische Schwingung einer Synchronmaschine behaftet ist, mit einiger Sicherheit voraus zu berechnen, weil zu viele Einflüsse in dieser einen Größe zusammentreffen.

Die Theorie des Parallelbetriebes ist also noch immer entwicklungsfähig. Um so wichtiger erscheint es mir, die gesicherten Grundlagen der Theorie festzuhalten, wie sie $z$. B. von Ossanna in dem Taschenbuch „Starkstromtechnik" ${ }^{3}$ ) in besonders

1) Wirklich einwandfrei wird man die Oberflächentemperatur bewegter Maschinenteile nur durch Messung bei der Bewegung feststellen können. Da Messungen wie die von E. Hinlein (Z. d. V. d. J. 55. S. 730. IgrI) natürlich nur an besonderen Versuchsmaschinen möglich sind, könnte man daran denken, die Temperatur bewegter Flächen durch Strahlungsmessung zu be. stimmen. Der Bau eines hierfür geeigneten praktischen Meßinstruments ist freilich keine einfache Aufgabe.

9) "Erzwungene Schwingungen bei veränderlicher Eigenfrequenz und ihre technische Bedeutung ${ }^{\prime \prime}$, Sammlung Vieweg, Heft $4^{\mathrm{r} / 42}$, rgr8.

3) Herausgegeben von Rziha und Seidener, Verlag Ernst v. Sohn. 